\title{
THE ESTIMATION OF EPISTASIS IN COMPONENTS OF FITNESS IN EXPERIMENTAL POPULATIONS OF DROSOPHILA MELANOGASTER I. A TWO-STAGE MAXIMUM LIKELIHOOD MODEL
}

\author{
A. G. CLARK, ${ }^{*}$ M. W. FELDMAN and F. B. CHRISTIANSEN \\ Department of Biological Sciences Stanford University Stanford, CA 94305
}

Received 15.x.80

\section{SUMMARY}

\begin{abstract}
Laboratory populations of Drosophila melanogaster bearing the Curly and Plum marked second chromosome inversions were observed in selection experiments for ten discrete generations. Maximum likelihood estimates of the relative fitnesses of Curly, Plum, Curly-Plum, and wild phenotypes were obtained from selection trajectories. Using these estimates, measures of multiplicative and additive epistasis were calculated. These were partitioned into pre-sampling and post-sampling components, and both were found to be significant. In several cases the sign of the epistasis of the two components was reversed, and the direction of net epistasis depended on the particular inversion. The significance of partitioning epistasis into components is discussed in the light of two locus population genetic theory.
\end{abstract}

\section{INTRODUCTION}

THE theory of selection when fitness is determined by two loci (Kimura, 1956; Lewontin and Kojima, 1960; Bodmer and Felsenstein, 1967; Karlin, 1975), emphasizes that evolution does not occur at single loci independently when epistatic interactions in fitness are present. These interactions may affect experimental evaluations of selective differences between genotypes identified by single electrophoretic loci. Inter-locus fitness interactions may also affect apparent population structure and allele frequency configurations of neutral loci, thereby invalidating commonly used tests of genetic distance and neutrality. It is therefore of considerable interest to experimentally assess the significance of fitness interactions.

Evidence of interlocus interactions in populations has for the most part been indirect. Since theory predicts that nonrandom associations between genes may be generated by epistasis, considerable effort has been devoted to the search for linkage disequilibrium between electrophoretically detected loci (Baker, 1975; Loukas and Krimbas, 1975; Zouros and Krimbas, 1973; Bodmer and Bodmer, 1978; Charlesworth et al., 1979). Despite the fact that strong linkage disequilibrium between loci was found to be rare, it is probably premature to suggest that epistasis is a rare phenomenon in nature. For example it is not clear how this picture will change as electrophoretically cryptic alleles are resolved (Weir and Cockerham 1978). Pairs of inversions, on the other hand, often occur in linkage disequilibrium (Levitan, 1955, 1958; Brncic, 1961; Mather, 1961; Stalker, 1960; Martin, 1962; Parsons,

* Present address: Dept. of Zoology, Arizona State University Tempe, AZ 85281 
1963; Lewontin and White, 1960), and inversions are often associated with particular electromorphs (Prakash and Lewontin, 1968, 1971; Prakash 1974, 1977; Loukas et al., 1979). None of these studies directly addresses the issue of the significance of epistasis, because convincing evidence that these disequilibria are generated by selective interactions is lacking. Alternative hypotheses such as founder effects, drift and migration have not been ruled out.

In laboratory populations of Drosophila melanogaster, Cannon (1963) and Birley (1974) observed significant linkage disequilibrium between visible markers, but failed to demonstrate fitness interactions. Clegg et al. (1980) observed a faster than expected decay in disequilibrium, while Clegg et al. (1978) reported a sustained nonrandom association between $P G M$ and $E s t-C$ after 40 generations. From these data they concluded that there was a nonuniform distribution of selective effects and interactions between loci. Barker (1977) observed that the relative fitness of white-eyed Drosophila melanogaster depended on the genetic background, implying either an interaction or an induced fitness effect. Similarly, Frydenberg (1963) found that the behaviour of a Stubble allele depended on its association with an inversion, suggesting that "associative overdominance" maintained the polymorphism.

Genes which transcribe enzymes of related function may interact phenotypically, as shown by Bijlsma (1978) in the case of G6PD and 6PGD, and studied by Marinkovic and Ayala $(1975 a, 1975 b)$ in Pgm-1, Me-2, Est-5, Odh and $M d h-2$. None of these studies measured fitness components with the intention of predicting population behaviour (see Prout $1971 b$ ), so the evolutionary significance of the measured interactions is not clear. Wilson $(1968,1972)$ studied fitness interactions by comparing fitness estimates of Drosophila melanogaster genotypes in selection experiments. Unfortunately the estimates were not all consistent, suggesting that uncontrolled or background genes caused selective differences.

Ideally experimental fitness estimates should be split into components of viability, fertility, gametic and sexual selection, and an experimental assessment of epistasis should be similarly partitioned. A pair of linked phenotypically dominant, recessive lethal genes is well suited for this purpose, since the number of dimensions is greatly reduced. Of the four epistatic parameters usually defined for the 9 two-locus phenotypes, we will in this system only consider that pertaining to the four surviving phenotypes. This epistasis can be simply defined as the deviation of the double heterozygote fitness from the expectation based on the single heterozygote fitnesses relative to wild type. For example, if the double heterozygote fitness deviates from the product of the single heterozygote fitnesses, there is nonzero multiplicative epistasis (Feldman and Crow, 1970). This paper analyzes net selective effects in trajectories of discrete generation experimental populations of Drosophila melanogaster. The components of twolocus selective values are further analyzed in a subsequent paper (Clark and Feldman, 1981a).

\section{MAterials AND MEthods}

Stocks of Oregon-R, Curly/Plum ${ }^{1}$ and $\mathrm{Plum}^{2} / \mathrm{mi}$ were obtained from the Bowling Green stock center. The second chromosome inversions 
bearing the markers $\mathrm{Cy}$ and $\mathrm{Pm}^{1}$ overlap, supressing recombination, whereas the inversion marked by $\mathrm{Pm}^{2}$ does not overlap with the $\mathrm{Cy}$ inversion. A range of crossover frequencies from $0.357 \pm 0.012$ to $0.395 \pm$ 0.013 was observed between the breakpoints of the Cy and $\mathrm{Pm}^{2}$ inversions, with the rate depending on the background and phase of the marked inversions (Clark and Feldman, 1981b).

All selection experiments were performed with two genetic backgrounds. In populations designated as "heterogeneous," the stocks were intercrossed for a number of generations to randomize the background. Since the fitness effects observed were due to large blocks of genes held relatively intact by inversions, the extent of randomization of the background was less than that necessary to demonstrate a single locus effect. The crossing protocol (fig. 1) assured a similarly randomized genetic background

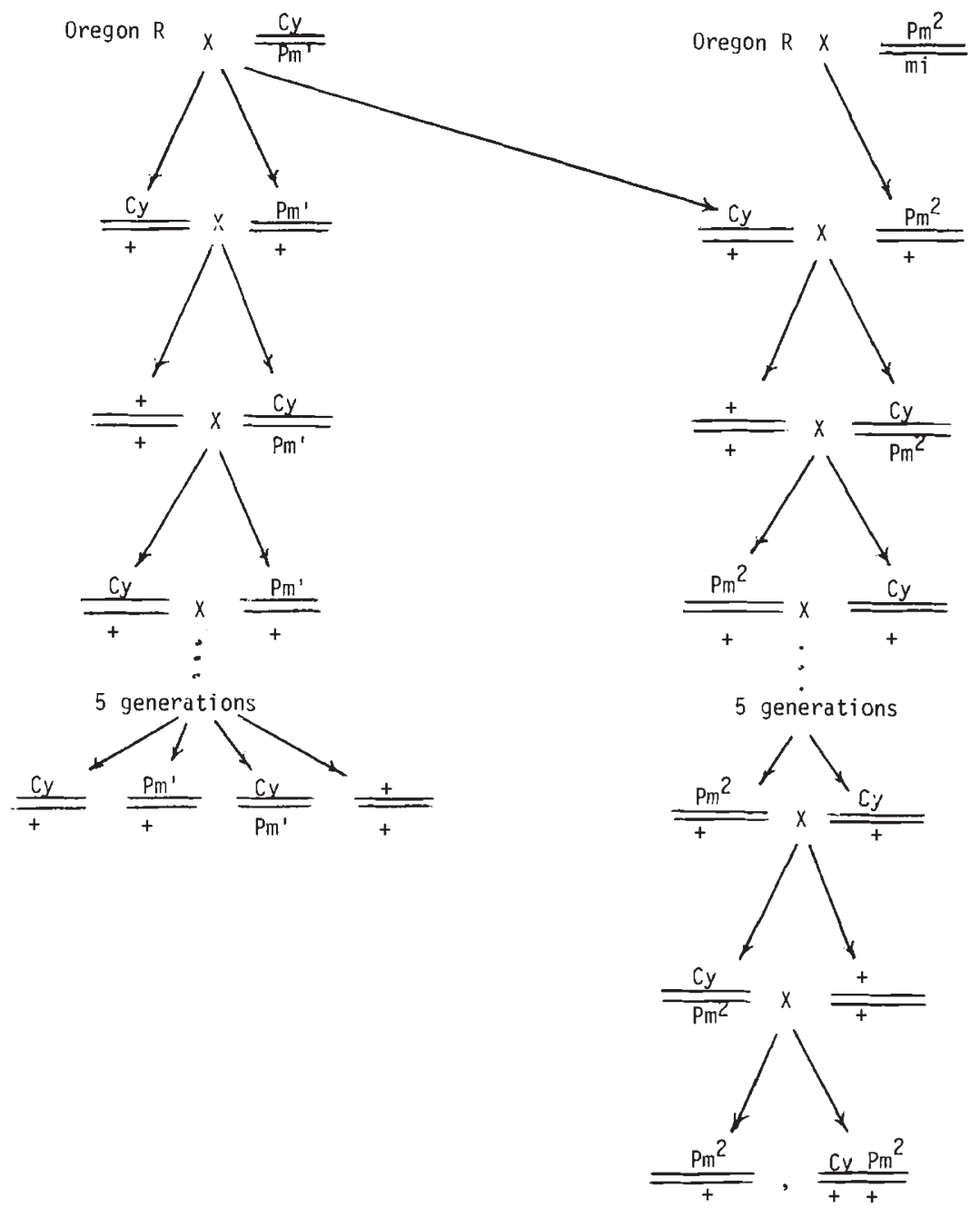

Fig. 1.-Crossing diagram for derivation of lines designated "heterogeneous". All stocks end up with the Oregon- $\mathrm{R}$ cytoplasm. The balancer mi (minus) is discarded. 
in all stocks, and all stocks had the Oregon-R cytoplasm. Stocks designated "homogeneous" were made isogenic for chromosome I and for regions spanned by the inversion on chromosome II (see fig. 2).

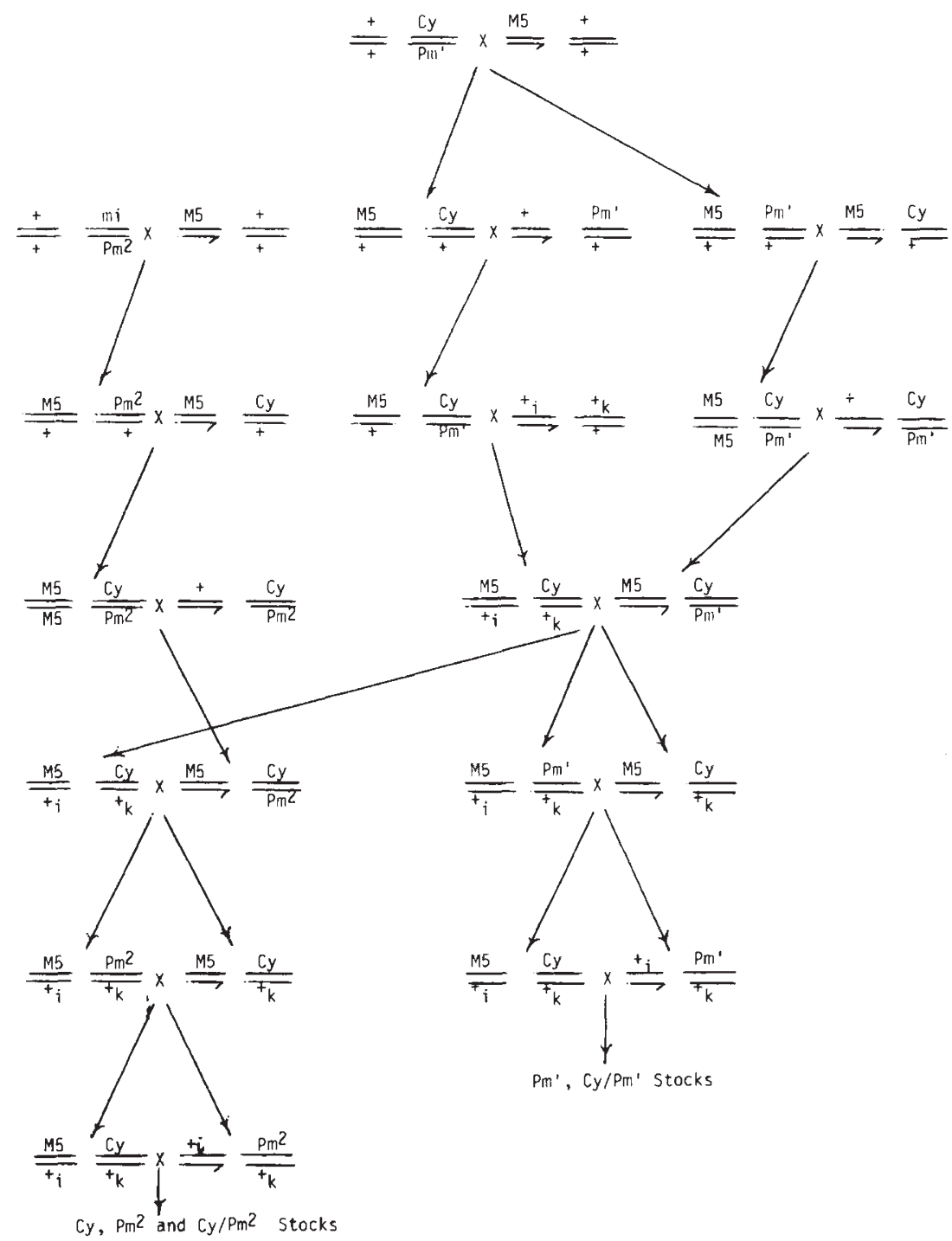

FIG. 2.-Crossing diagram for derivation of "homogeneous" lines. These all share a wild second chromosome and are homozygous X. M5 is the Muller-5 marked balancer X chromosome.

Six population configurations were initialized with 6 different phenotype frequencies (table 1). The three populations designated $\mathrm{Cy}, \mathrm{Pm}^{1}$ and $\mathrm{Pm}^{2}$ each had only one inversion segregating and will be referred to as marginal. The $\mathrm{Cy} / \mathrm{Pm}^{\mathrm{T}}$ population had the $\mathrm{Cy}$ and $\mathrm{Pm}^{1}$ inversions segregating, but since these do not recombine, the populations behaved as a 1 locus 3 allele 
TABLE 1

Initial genotype frequencies for the six selection experiments. Four replicates of each treatment with two different genetic backgrounds were studied (a total of 48 populations). Populations were started with equal numbers of 4 day old virgins of each sex in half-pint bottles. Note that $\mathrm{Pm}^{l}$ and $\mathrm{Pm}^{2}$ never appear in the same population

$\begin{array}{cccccccc}\text { Population } & \mathrm{Cy} & \mathrm{Pm}^{1} & \mathrm{Pm}^{2} & \mathrm{Cy} / \mathrm{Pm}^{1} & \mathrm{Cy} / \mathrm{Pm}^{2} & \mathrm{Cy}-\mathrm{Pm}^{2} & \text { Wild } \\ \begin{array}{c}\mathrm{Cy} \\ \mathrm{Pm}^{1}\end{array} & 200 & & & & & & \\ \mathrm{Pm}^{2} & & 200 & & & & & \\ \mathrm{Cy} / \mathrm{Pm}^{1} & & & 200 & & & & 20 \\ \mathrm{Cy} / \mathrm{Pm}^{2} & & & & 180 & & & 20 \\ \mathrm{Cy}-\mathrm{Pm}^{2} & & & & & & 180 & 20\end{array}$

system. The $\mathrm{Cy} / \mathrm{Pm}^{2}$ and $\mathrm{Cy}-\mathrm{Pm}^{2}$ populations were analogous to populations with 2 diallelic loci segregating, initially in strong negative and positive linkage disequilibrium respectively.

Populations were maintained on an 18 day discrete generation schedule. On day 0 populations were started with 4 day old virgins in the phenotype frequencies shown in table 1 . All bottles had equal numbers of males and females and an initial adult density of 200 flies. On day 6 the adults were removed and discarded. On day 18 the adult progeny were anesthetized and scored by phenotype (the genotypes $\mathrm{Cy}+/+\mathrm{Pm}^{2}$ and $\mathrm{Cy}-\mathrm{Pm}^{2} /++$ could not be distinguished). Fresh bottles were innoculated with these adults in the observed frequencies and with a total density of 200 .

All populations were raised in half-pint bottles on Carolina 4-24 instant Drosophila medium ( $12 \mathrm{~g}$ medium, $35 \mathrm{ml}$ water and a dash of live yeast per bottle). All bottles were maintained in an incubator with a 12 hour day/12 hour night cycle at $25 \pm 1{ }^{\circ} \mathrm{C}$. Four replicates of each of 6 initial conditions and 2 genetic backgrounds (for a total of 48 populations) were followed for 10 generations.

\section{(i) One locus fitness estimation}

Estimates of the relative fitness of phenotypes were made by statistically fitting models to the phenotype frequency trajectories. The model used to determine relative fitnesses in the marginal populations is due to Prout (1965) and Anderson (1969). This model assumes that mating occurs randomly, that the two sexes have the same fitness, and that fitness parameters are constant. Fitness is split into two temporal components to avoid problems of apparent frequency dependence (Prout 1965, 1969). The early component $(E)$ indicates the relative fitness from zygote to the time of adult sampling. The late component $(L)$ represents the fitness from population initiation to zygote formation, and therefore includes some adult mortality as well as fertility effects. In all cases the wild type flies were assigned an early component $(E=1)$, and a late component $(L=1)$. Net fitness is the product of the two temporal components $(W=E \times L)$. Although the model does not distinguish effects of viability, fertility, and sexual selection, it gives a reasonable estimate for net fitness. Its statistical power in separating the net fitness into an $E$ and $L$ component is fairly weak (see Discussion). This was reflected in the between replicate heterogeneities, which were high for the $E$ and $L$ components, but consistently lower in 
the net fitnesses. For this reason, we place emphasis on the net fitness estimates. Expected phenotype frequencies were calculated by weighting observed phenotype frequencies by the late components, generating expected zygote frequencies by random mating, and weighting the zygote frequencies by the $E$ components. The expected trajectories were thereby calculated one generation at a time based on observed phenotype frequencies of the preceding generation. This method deviates from previous studies (Prout, 1969; Anderson, 1969; and Wilson, 1968) which generated expected trajectories for all generations based on fitness estimates and the initial population composition. Statistical advantages of our method will be discussed below. The model was fitted to the data for each of four replicates separately by minimizing the $G$ statistic $(-2 \times \log$-likelihood ratio) with respect to $E$ and $L$. These $G$ values are designated $G_{1}, G_{2}, G_{3}$, and $G_{4}$ in the tables. The model was also fitted jointly to the four replicates of each experiment giving an estimate of $E$ and $L$ which minimized the total $G$. The heterogeneity between replicates was then assessed by evaluating $G($ het $)=$ $G$ (total) $-G_{1}-G_{2}-G_{3}-G_{4}$ as a chisquare test statistic.

\section{(ii) Two locus fitness estimation}

An extension of the Prout model was used to estimate the fitnesses of the Curly, Plum and Curly/Plum phenotypes relative to the wild type based on trajectories in population bottles. The net fitness was again split into two temporal components for each phenotype (table 2). Depending on the

\section{TABLE 2}

Definition of fitness parameters. Net fitness is defined as $W_{1}=E_{1} \times L_{1} . E_{2}$ and $L_{2}$ designate fitness of either $\mathrm{Pm}^{1}$ or $\mathrm{Pm}^{2}$ depending on the population. Note that we assume no position effect in fitness

$\begin{array}{ccc}\text { Genotype } & \text { Early component } & \text { Late component } \\ \text { Cy } & E_{1} & L_{1} \\ \mathrm{Pm} & E_{2} & L_{2} \\ \mathrm{Cy} / \mathrm{Pm} & E_{3} & L_{3} \\ \text { Cy-Pm } & E_{3} & L_{3} \\ \text { Wild } & 1 \cdot 0 & 1 \cdot 0\end{array}$

population, $E_{2}$ and $L_{2}$ represent fitness estimators for $\mathrm{Pm}^{1}$ or $\mathrm{Pm}^{2}$, and $E_{3}$ and $L_{3}$ correspond to either $\mathrm{Cy} / \mathrm{Pm}^{1}$ or $\mathrm{Cy} / \mathrm{Pm}^{2}$ and $\mathrm{Cy}-\mathrm{Pm}^{2}$ genotypes. Since the two double heterozygotes bearing $\mathrm{Cy}$ and $\mathrm{Pm}^{2}$ cannot be distinguished phenotypically, it is not possible to estimate their relative fitness from only phenotype trajectories. For this reason we assume that there is no position effect in fitness. As in the one locus model, we assume constant fitness, random mating, no sex differences in fitness, and multinomial population sampling.

The expected trajectories were calculated recursively as in the one locus model (fig. 3). For each set of $E_{i}$ and $L_{i}$, a ten generation trajectory of linkage disequilibrium values, $D(t)$, was calculated based on the initial population composition. The four observed phenotype frequencies at generation $t\left(p_{1}, p_{2}, p_{(3+4)}, p_{5}\right.$ for $\mathrm{Cy}, \mathrm{Pm}, \mathrm{CyPm}$ and wild respectively) were used to estimate a vector of five genotype frequencies $\left(g_{1}, g_{2}, g_{3}, g_{4}, g_{5}\right)$ 


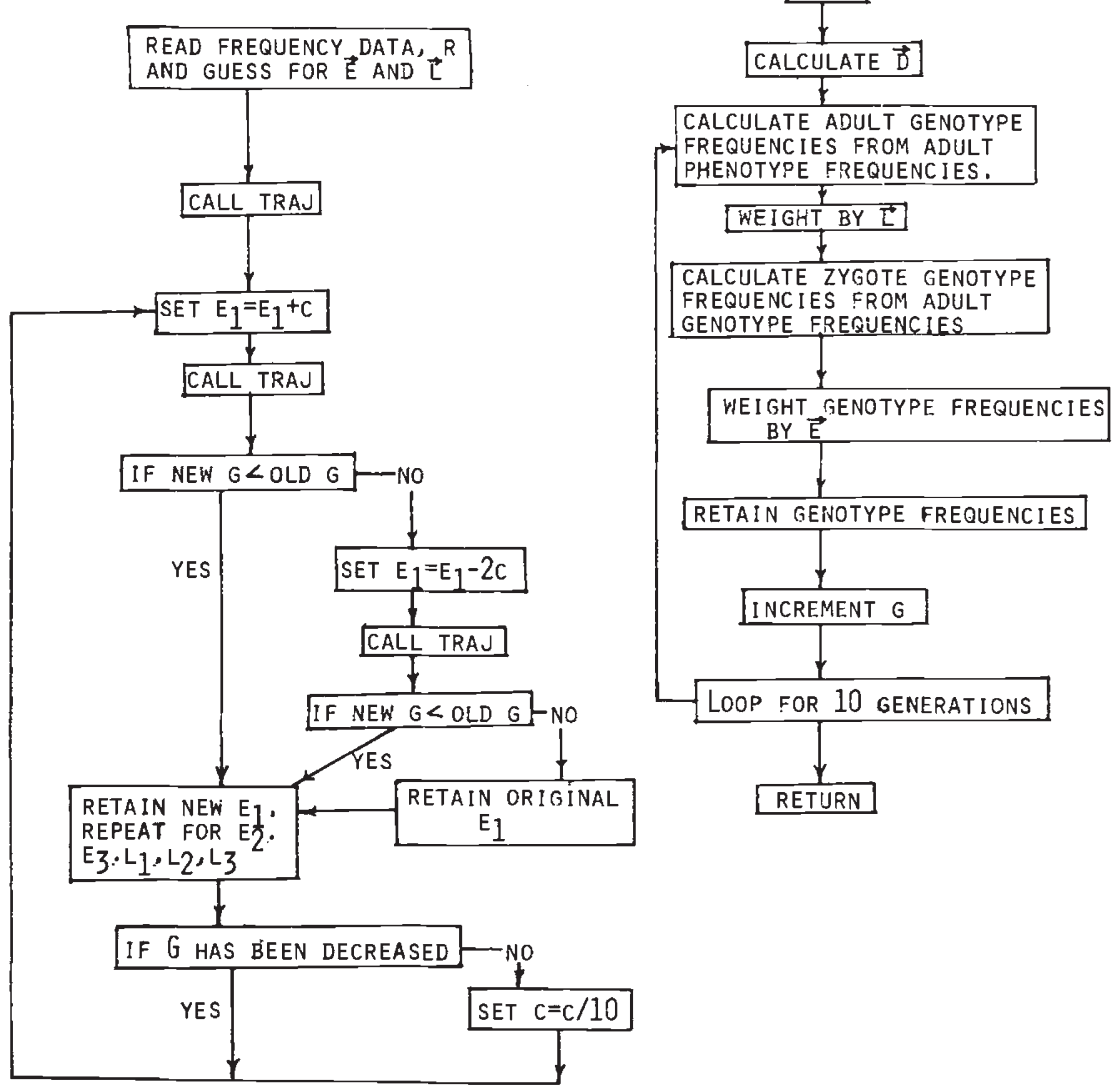

FIG. 3.-Flow diagram for the maximum likelihood two locus fitness estimating program. Iteration terminates when the goodness of fit plateaus.

based on the linkage disequilibrium values predicted by the model, i.e., $g_{i}=p_{i}$ for $i=1,2,5, \quad g_{3}=\left[p_{(3+4)}+D(t)\right] / 2$ (trans phase) and $g_{4}=$ $\left[p_{(3+4)}-D(t)\right] / 2$ (cis phase). These 5 genotype frequencies are weighted by their respective late fitness parameters, i.e., $x_{i}=L_{i} g_{i} / \bar{L}$. The following equations then give the genotype frequencies among zygotes after elimination of lethal genotypes at the start of generation $t+1$ :

$$
\begin{aligned}
T x_{1}{ }^{\prime}= & x_{1}\left(x_{1}+x_{2}+x_{3}+x_{4}+2 x_{5}\right)+x_{3}\left(x_{2}+x_{4}+2 x_{5}\right) \\
& -0 \cdot 5 r\left[\left(x_{3}-x_{4}\right)\left(x_{2}-x_{3}+x_{4}+2 x_{5}\right)\right] \\
T x_{2}{ }^{\prime}= & x_{2}\left(x_{1}+x_{2}+x_{3}+x_{4}+2 x_{5}\right)+x_{3}\left(x_{1}+x_{4}+2 x_{5}\right) \\
& -0 \cdot 5 r\left[\left(x_{3}-x_{4}\right)\left(x_{1}-x_{3}+x_{4}+2 x_{5}\right)\right] \\
T x_{3}{ }^{\prime}= & x_{3}\left(x_{1}+x_{2}+x_{3}\right)+x_{1} x_{2}-0 \cdot 5 r\left[\left(x_{3}-x_{4}\right)\left(x_{1}+x_{2}+2 x_{3}\right)\right] \\
T x_{4}{ }^{\prime}= & x_{4}\left(x_{1}+x_{2}+x_{4}+2 x_{5}\right)+0 \cdot 5 r\left[\left(x_{3}-x_{4}\right)\left(x_{1}+x_{2}+2 x_{4}+2 x_{5}\right)\right] \\
T x_{5}{ }^{\prime}= & x_{1}\left(0 \cdot 5 x_{1}+x_{2}+x_{4}+2 x_{5}\right)+x_{2}\left(0 \cdot 5 x_{2}+x_{4}+2 x_{5}\right)+x_{4}\left(0 \cdot 5 x_{4}+2 x_{5}\right) \\
& +2 x_{5} x_{5}+0 \cdot 5 r\left[\left(x_{3}-x_{4}\right)\left(x_{1}+x_{2}+x_{4}+2 x_{5}\right)\right]
\end{aligned}
$$


where $x_{1}, x_{2}, x_{3}, x_{4}$ and $x_{5}$ were the frequencies of Curly, Plum, Curly/Plum, Curly-Plum and wild respectively, and $T$ is the normalizer to make the frequencies sum to one. The $\mathrm{Cy} / \mathrm{Pm}^{1}$ populations have no coupling double heterozygotes so $x_{4}=0$. This set of genotype frequencies is then weighted by the early components $\left(x_{i}{ }^{\prime \prime}=E_{i} x_{i}{ }^{\prime} / E\right)$. The vector of genotype frequencies at this point is stored, and represents a population sampled after the $E$ selection. Phenotype frequencies are then calculated from these predicted genotype frequencies, and the $G$ statistic is incremented using the predicted and observed phenotype frequencies at generation $t+1$. This procedure is repeated for 10 generations in each of the four replicates.

The goodness of fit of the model's predicted trajectory, based on the estimated values of $E_{i}$ and $L_{i}$, to the observed trajectory is determined using the $G$ statistic. Minimizing the $G$ statistic is equivalent to maximizing the log-likelihood ratio. The $G$ statistic has the advantage that it can be

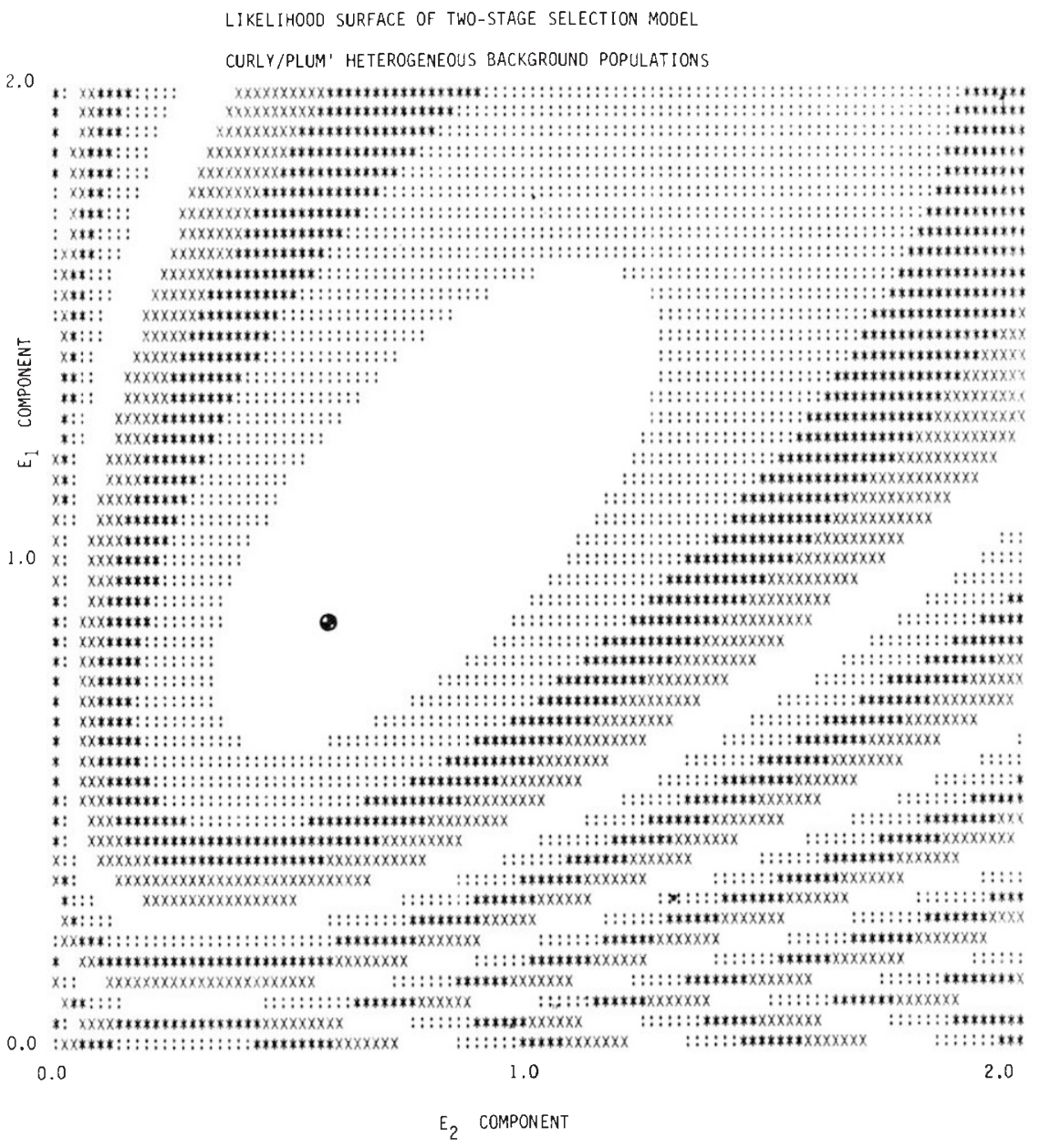

FIG. 4.-Contour map of a two dimensional slice of the homogeneous Cy-Pm2 population likelihood surface. Symbols represent different values, of $G$ for each point in the $E 1-E 2$ plane when other parameters are fixed. The likelihood maximum is indicated by the dot. 
partitioned into a measure of heterogeneity over replicates and a net goodness of fit. The program tests different values of the fitness estimators and only accepts those that decrease the total $G$. Although this method is slower than Fisher's scoring technique, it can never diverge from a likelihood maximum.

Two methods were employed to guarantee that the likelihood function had a unique maximum. Iterations were started from different sets of $E$ and $L$ values, and all converged to the same maximum. The likelihood surfaces were also plotted as contour maps which clearly showed a unique maximum for each parameter (fig. 4). The program was tested in two ways. First, it was used to estimate fitnesses in the marginal populations and yielded the
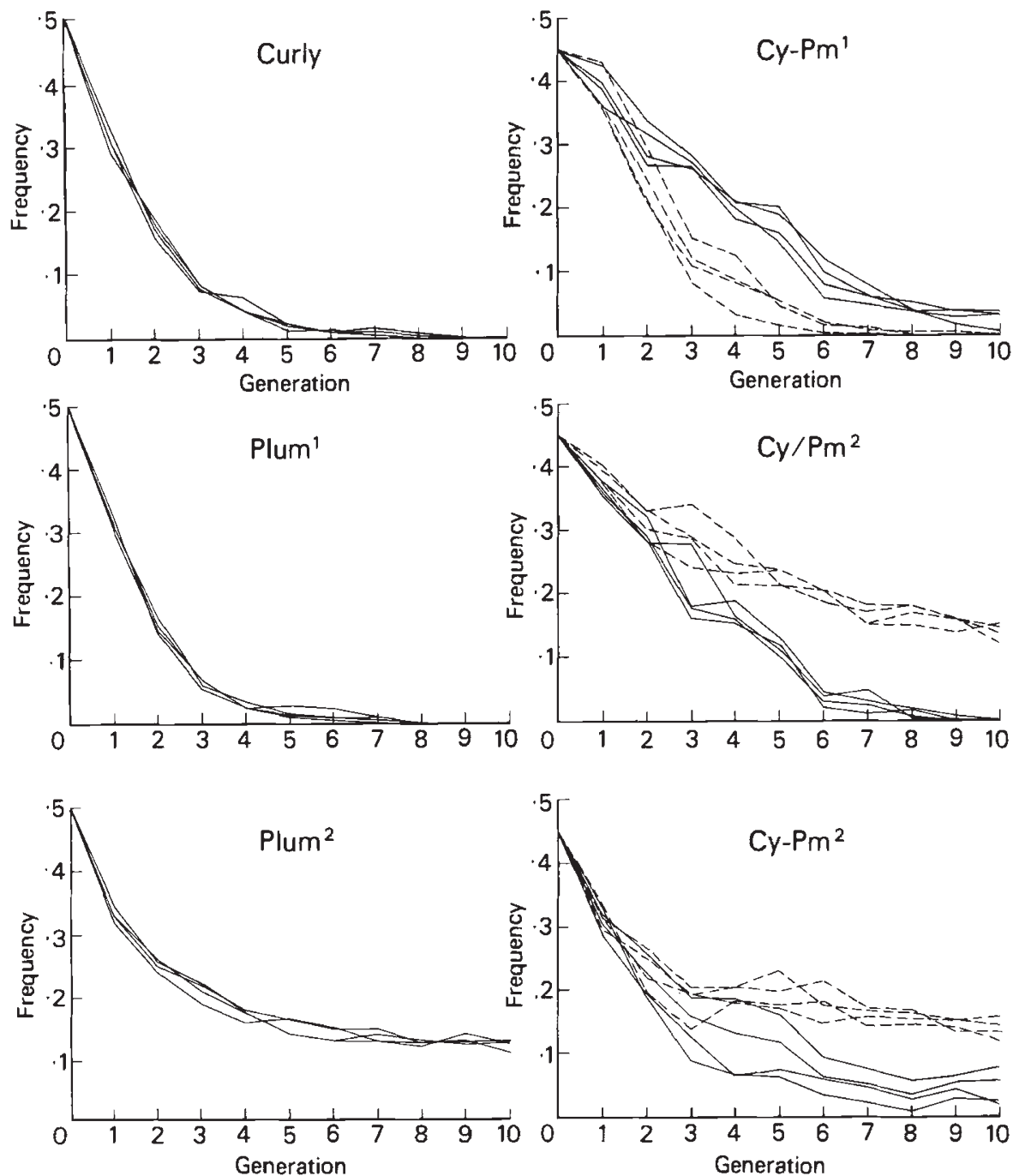

FIG. 5.-Trajectories of the dominant marker allele frequencies in populations with hetero* geneous background. Dashed lines represent Plum frequencies, while solid lines indicate Curiy frequencies. 
same estimators as the one locus model. Second, several sets of trajectories were calculated deterministically using arbitrary fitnesses. When these trajectories were presented as data to the maximum likelihood routine, it did in fact converge to the expected fitness values.

\section{Results}

Graphs of the $\mathrm{Cy}$ and $\mathrm{Pm}$ phenotype frequencies in the marginal populations are presented in the left half of fig. 5 and 6 . Since both Cy and $\mathrm{Pm}$ are lethal when homozygous, the only remaining genotypes in the marginal populations are either wild type or heterozygotes. If their fitnesses
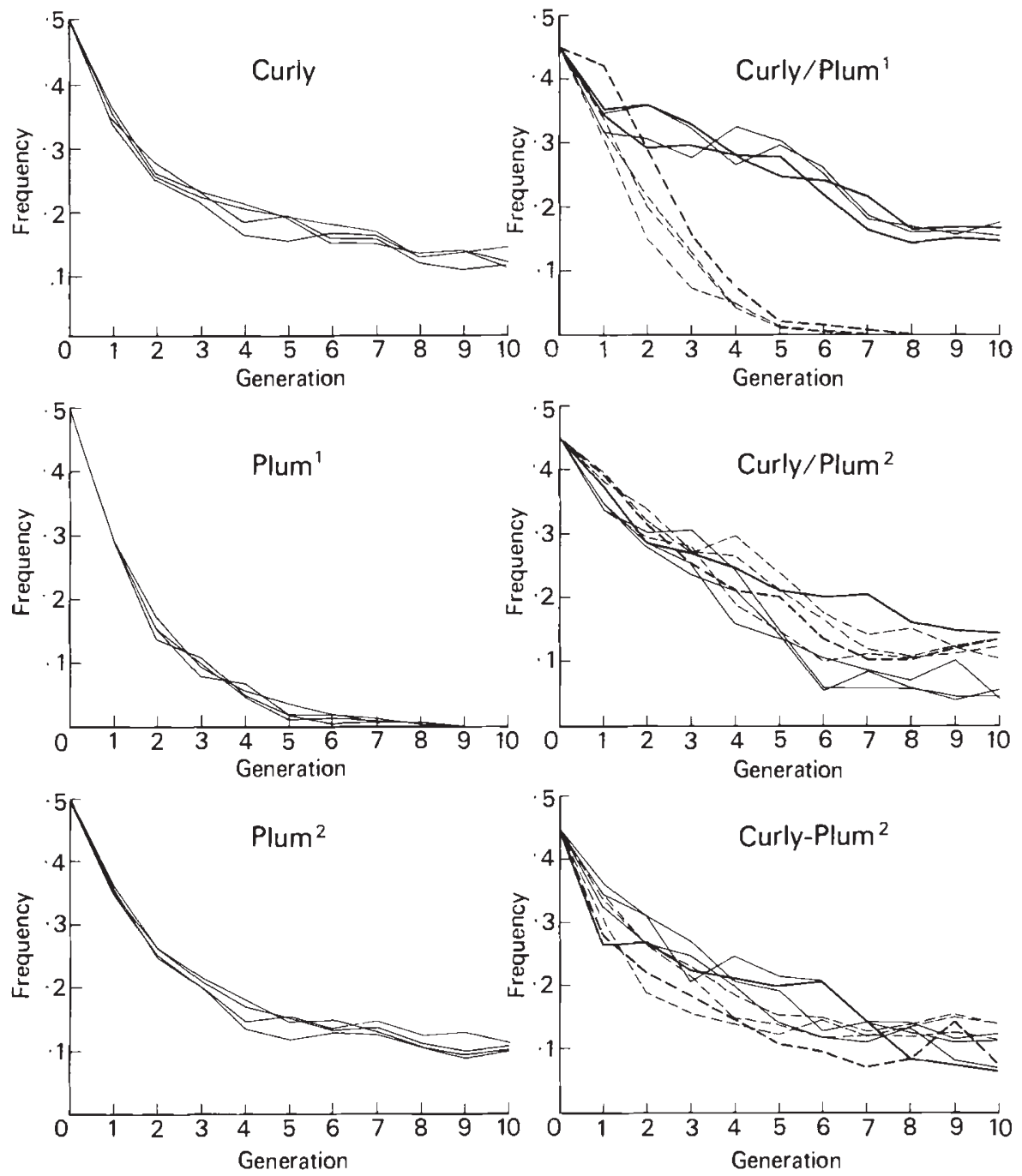

Fig. 6.-Trajectories of the $\mathrm{Cy}$ and $\mathrm{Pm}$ allele frequencies in the populations with homogeneous background. Dashed lines represent Plum frequencies, while solid lines indicate Curly frequencies. 
were equal, then the allele frequencies would follow the lethal trajectory, $p(t)=1 /(2+t)$. By inspection, the Cy and $\mathrm{Pm}^{1}$ trajectories with heterogeneous background fall below this theoretical curve, indicating a heterozygote disadvantage. The $\mathrm{Pm}^{2}$ trajectories on the other hand lie slightly above the lethal curve, and in fact appear to be approaching a stable equilibrium. In fig. 6 when the background is homogeneous, the $\mathrm{Pm}^{1}$ trajectory is essentially the same as in fig. 5 , but both the Cy and $\mathrm{Pm}^{2}$ are now maintained. This in itself is a demonstration of an associative fitnesss effect between the genes on the Cy chromosome and the background genotype.

Trajectories of allele frequencies in the populations which had both $\mathrm{Cy}$ and $\mathrm{Pm}$ segregating also appear in fig. 5 and 6 . The qualitative result is as expected based on the marginals. In the heterogeneous populations, the $\mathrm{Cy}$ and $\mathrm{Pm}^{1}$ alleles are lost, while the $\mathrm{Pm}^{2}$ attains an equilibrium frequency about the same as in the marginal. The homogeneous populations also behave as the marginals, with $\mathrm{Cy}$ and $\mathrm{Pm}^{2}$ persisting in all populations, and $\mathrm{Pm}^{1}$ being lost. An example of the phenotype trajectories is given in fig. 7

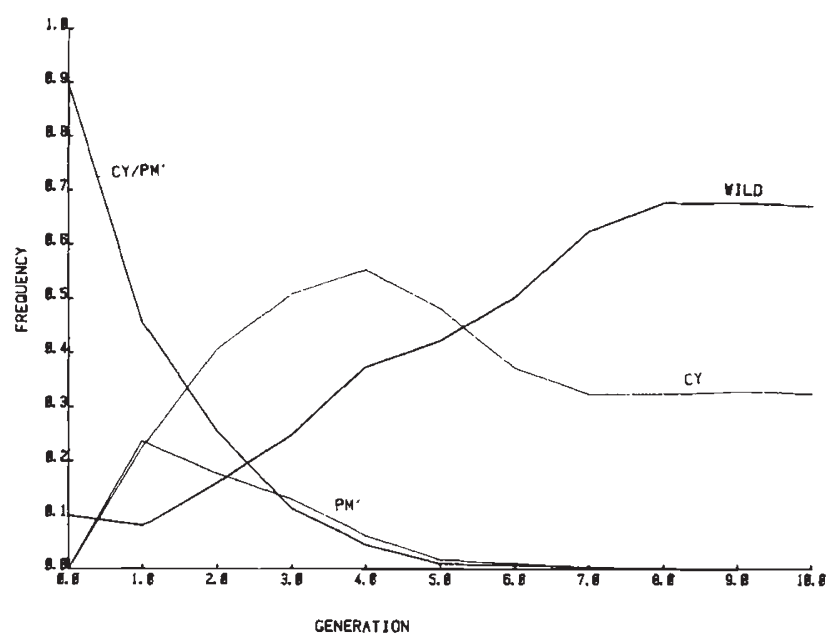

FIG. 7.-Phenotype trajectories of the pooled $\mathrm{Cy} / \mathrm{Pm} 1$ populations with homogeneous background.

for the $\mathrm{Cy} / \mathrm{Pm}^{1}$ populations with homogeneous background. Note that as the $\mathrm{Cy} / \mathrm{Pm}^{1}$ phenotype declines in frequency, the breakdown of the strong initial association generates an excess of $\mathrm{Cy}$ and $\mathrm{Pm}^{1}$ phenotypes. As the association weakens, these phenotypes then decline in frequency in favour of wild types.

The goodness of fit of the model can be assessed by observing the correspondence between the observed and predicted trajectories. One hundred trajectories were generated for each of the six selection treatments by iterating the recursions and weighting by estimated fitness components. At the adult stage in each generation there was a multinomial sampling with a sample size equal to the mean number of adults observed in the experimental populations in the respective generation. The empirical ninety-six per cent confidence regions were represented by plotting the third and 


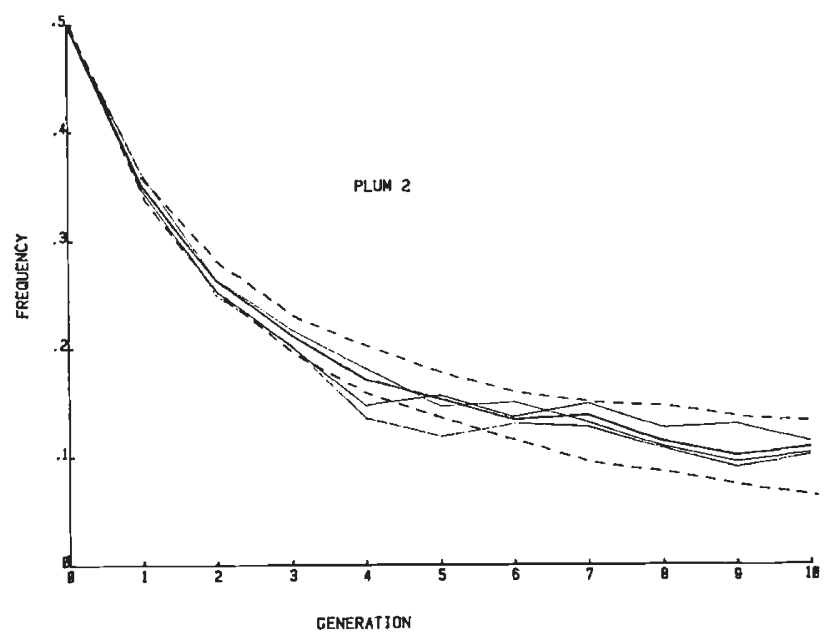

FIG. 8.-Trajectories of homogeneous $\mathrm{Pm} 2$ populations with the confidence region based on the maximum likelihood fitness estimates superimposed. The four replicates are plotted in solid lines, while the predicted confidence region is dashed. The confidence region is constructed by generating 100 trajectories based on the estimated fitnesses and performing a multinomial sampling of adults each generation. Ninety-six of these curves fall within the region plotted.

ninety-eighth highest allele frequency at each generation. The size of these confidence regions is due to sampling error alone, and temporal variation in fitness or environmental fluctuation would further increase the spread. Fig. 8 and 9 are representative examples.

The $G$ statistics are recorded for the marginal populations in table 3. In all 6 cases the total $G$ value is less than the critical value, indicating an acceptable fit. Furthermore, no case shows significant heterogeneity

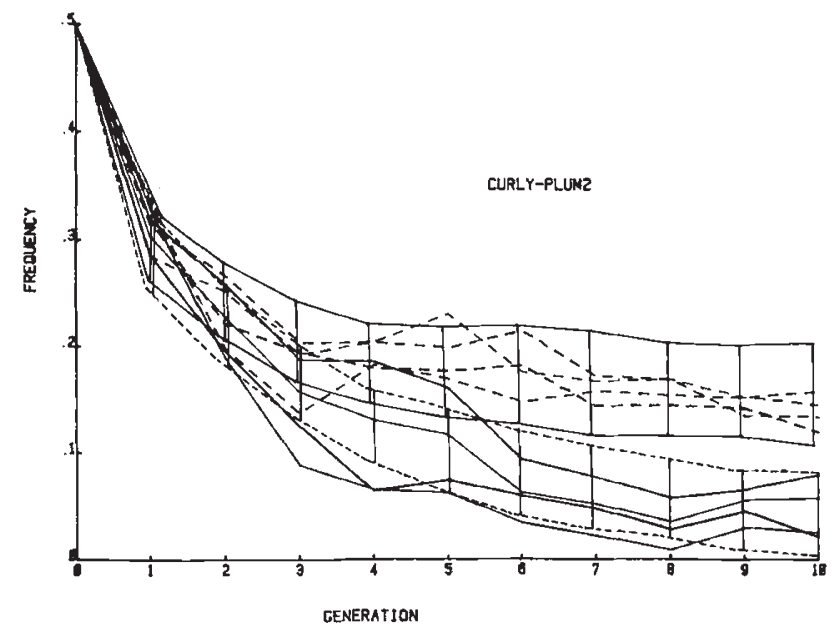

FIG. 9.-Trajectories of the heterogeneous Cy-Pm2 populations with the confidence regions. The solid lines fit the $\mathrm{Cv}$ allele trajectory, and the dashed lines fit the Pm trajectory. The four replicate $\mathrm{Cy}$ allele frequencies are plotted with dashed lines, while the $\mathrm{Pm} 2$ allele frequencies are plotted with solid lines. Confidence regions were constructed as in fig. 8 . 
FITNESS IN DROSOPHILA

(2)

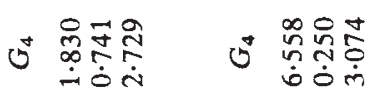

O

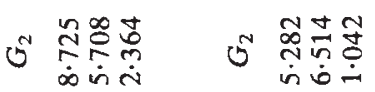

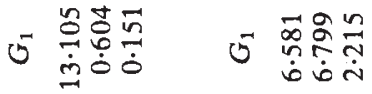

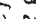

ปs

苋

ริ

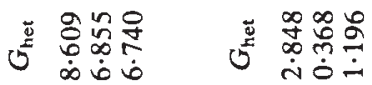

吝

is

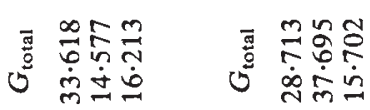

望

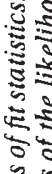

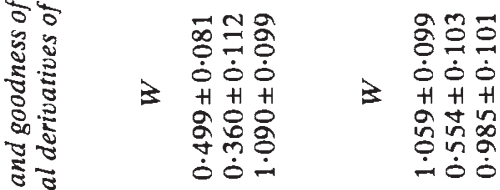

蛋

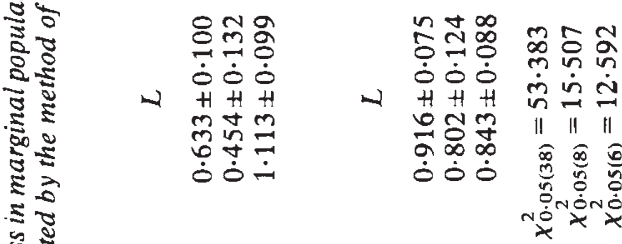

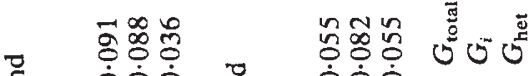

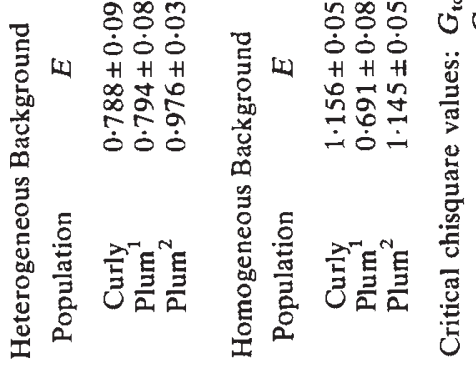


between replicates. Of the 24 marginal trajectories, 23 are adequately explained by the model at the 5 per cent level of significance. The values of the fitness estimators are in qualitative agreement with expectations based on the graphs. With a heterogeneous background the $\mathrm{Cy}$ and $\mathrm{Pm}^{1}$ have a net fitness less than the wild type, while the estimated fitness of $\mathrm{Pm}^{2}$ was larger than one, suggesting overdominance. The Curly chromosome appears to be overdominant in the homogeneous background, confirming the interaction mentioned earlier. In both cases the degree of overdominance does not appear to be significant, although from Figures 5 and 6 there appears to be an approach to polymorphic equilibria.

Table 4 lists the fitness estimators for the populations with both $\mathrm{Cy}$ and $\mathrm{Pm}$ segregating. Of the 24 replicates independently tested, 18 gave adequate fits to the model. The total $G$ is obtained by summing $G$ values over replicates for the single best fitting set of $E_{i}$ and $L_{i}$. The heterogeneity $G$ value is obtained by subtracting the four $G$ values for the best fit to each replicate from the total $G$. By this criterion, the experiments show significant heterogeneity between replicates.

The similarity of fitness estimates in the $\mathrm{Cy} / \mathrm{Pm}^{2}$ and $\mathrm{Cy}-\mathrm{Pm}^{2}$ populations (table 4) suggested that these data sets be pooled. When this was done, the $G$ value for the best fit of the model to the pooled $\mathrm{Cy} / \mathrm{Pm}^{2}$ and $\mathrm{Cy}-\mathrm{Pm}^{2}$ data sets with heterogeneous background was 559.737 . Subtracting the $G$ (total) values for these two populations separately $(262.266$ and $294 \cdot 852$ ) gave a heterogeneity $G$ of $2 \cdot 619$, which was not significant $\left(\chi_{[0.05] 6 \text { d.f. }}^{2}=12 \cdot 592\right)$. In the heterogeneous background experiments, we can conclude that the two different starting conditions gave remarkably consistent fitness estimates. In contrast, the homogeneous background $\mathrm{Cy} / \mathrm{Pm}^{2}$ and $\mathrm{Cy}-\mathrm{Pm}^{2}$ populations yielded significantly heterogeneous fitnesses $\left(G_{\text {het }}=63.445\right)$, but this is on the same order as the heterogeneity between replicates within a treatment.

The data were also analyzed by using an extension of the method of Prout (1969) and Anderson (1969), wherein the expected trajectory was calculated for all ten generations based on the initial conditions and the fitnesses. Although the fitness estimates obtained by this method were quite similar to those obtained by the method reported here, our method gave substantially lower values of heterogeneity $G$. Using the classical method, we estimated fitnesses by pooling the data over replicates, and in all cases the pooled data gave adequate fits to the model. The classical method is inferior when estimating fitnesses jointly, because chance divergence between replicates in early generations artificially inflates the heterogeneity.

The machinery developed so far enables us to investigate epistasis in fitness. With the fitness estimates in hand, we can examine two measures of epistasis. As stated in the Introduction, a linked lethal system will have just one multiplicative and one additive measure of epistasis. In the notation given in table 2, the net additive epistasis and multiplicative epistasis measures are:

$$
\begin{aligned}
E(\text { net }, \text { add }) & =1-W_{1}-W_{2}+W_{3} \\
E(\text { net, mult }) & =W_{3} /\left(W_{1} \times W_{2}\right)-1
\end{aligned}
$$

respectively, where $W_{i}=E_{i}+L_{i}$. We can also estimate the epistasis in early and late components separately, e.g., the early epistasis measures are 


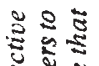

ธั้

है

ริ ปั

ㅎํㅇ

ริำ

월정

ป

उ.

\&

ธे

政

క

要范范

ट

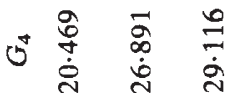

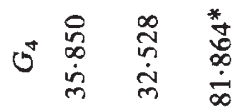

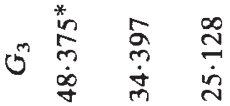

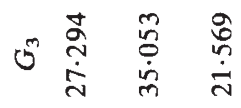

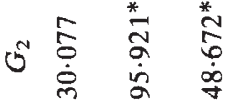

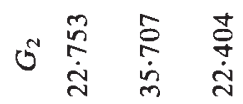

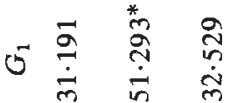

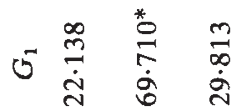

ํํำ

政

동

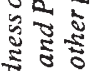

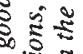

จ

o.

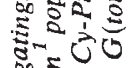

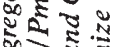

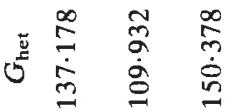

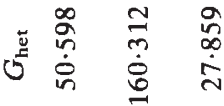

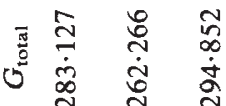

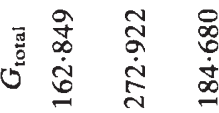

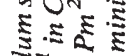

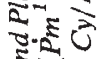

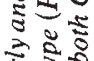

5

$\infty$ ह

कह

证変

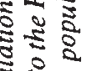

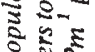

हैक

$\approx \Sigma$

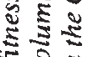

政:

है

ซँ

$\checkmark \dot{s}$

से

닌

氙密

ㅊำ

है

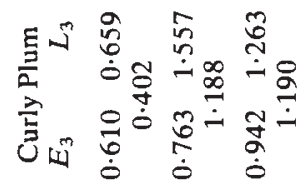

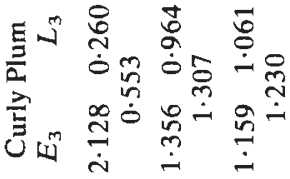

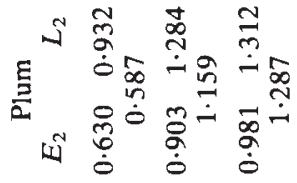

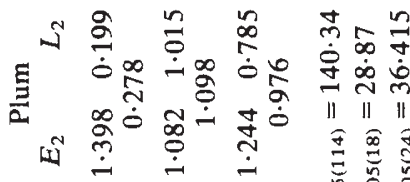

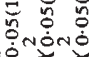

讦叫焉

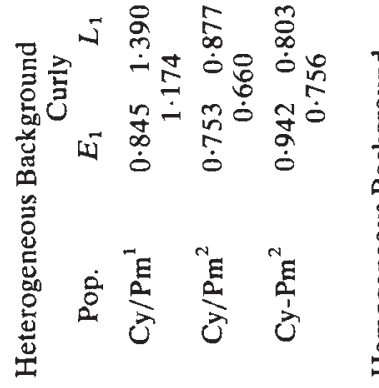

ت

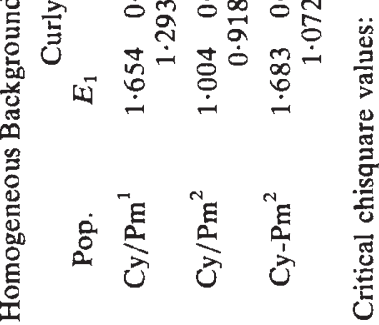


defined as:

$$
\begin{aligned}
E(\text { early }, \text { add }) & =1-E_{1}-E_{2}+E_{3} \\
E(\text { early }, \text { mult }) & =E_{3} /\left(E_{1} \times E_{2}\right)-1 .
\end{aligned}
$$

With these definitions, a positive epistasis indicates a double heterozygote fitness that is greater than the simple sum or product of single heterozygote fitnesses. The early and late multiplicative epistasis parameters can be multiplied to yield the net multiplicative epistasis:

$$
E(\text { net }, \text { mult })+1=[E(\text { early, mult })+1] \times[E(\text { late }, \text { mult })+1],
$$

whereas the net additive epistasis cannot be decomposed in this fashion. Zero multiplicative epistasis in the early and late components implies zero net multiplicative epistasis, but this is not true of the additive parameter.

Using the estimated fitness values these epistasis measures are tabulated in table 5. With both backgrounds, the $\mathrm{Cy}$ and $\mathrm{Pm}^{1}$ chromosome show negative additive epistasis, indicating a low fitness of double inversion carriers. In most other cases the net epistasis is positive. In 18 out of 24 replicate populations the signs of the multiplicative epistasis in the early and late components are reversed, but we cannot exclude the possibility that this is merely a statistical artifact. The heterogeneity observed between replicates (table 4) is reflected in the epistasis measures. That the early and late components of epistasis vary widely in both sign and magnitude betiveen replicates, suggests that the model fails to adequately partition the components.

The distribution of epistasis measures is not known, so standard errors are not reported in table 5, and parametric tests of significance cannot be applied. Rather than determining this distribution, another approach was used to test the significance of the fitness interaction. The estimation routine was changed in such a way that $E_{3}$ and $L_{3}$ were not allowed to vary freely, but were determined by the other four parameters. In the test for zero additive epistasis, $E_{3}$ was set equal to $E_{1}+E_{2}-1$ and $L_{3}=L_{1}+L_{2}-1$. As table 6 indicates, the zero epistasis model gave acceptable fits in just 7 out of 24 replicates. Likewise the zero multiplicative epistasis model set $E_{3}=$ $E_{1} \times E_{2}$ and $L_{3}=L_{1} \times L_{2}$. In this case, as seen in table 7, 6 out of 24 replicates adequately fitted the zero multiplicative epistasis model. The significance of the difference in goodness of fit was tested in two ways. When a $G$ value from the non-epistatic model is divided by a corresponding $G$ value from the epistatic model, and weighted by the appropriate degrees of freedom, an $F$ statistic is obtained. In the additive case, 20 out of 24 replicate populations yield $F$ values greater than $1 \cdot 0$, indicating that the non-epistatic model gives a worse fit. In the multiplicative case, 23 out of 24 values of $F$ are greater than 1.0 . Since we expect an $F$ value of 1.0 in the absence of epistasis, we can test this null hypothesis with a sign test on the observed $F$ statistics. In both the additive and the multiplicative cases, we conclude that epistasis increases the observed variance. In addition, if we sum the $24 G$ values for the non-epistatic model, and divide by the sum of the $24 G$ values for the epistatic model (weighting again by the degrees of freedom), we obtain a pooled test statistic that is approximately $F$ distributed. In the zero additive and multiplicative cases, these values are $1 \cdot 274$ and 1.351 respectively, where $F_{(624,576)[0.05]}=1 \cdot 089$. These findings 
FITNESS IN DROSOPHILA

(1)

Uे

๕ 눈

$3^{m}$

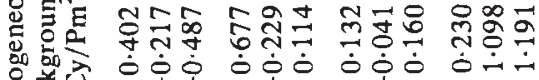

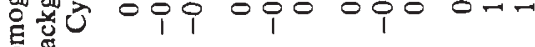
选 U

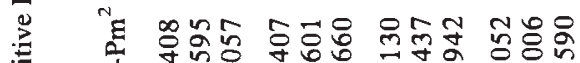

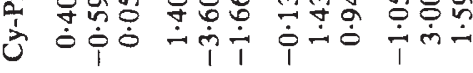
产 敬 造解 T乐

E

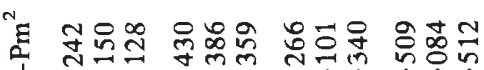

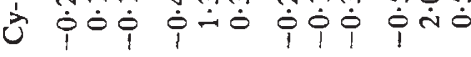

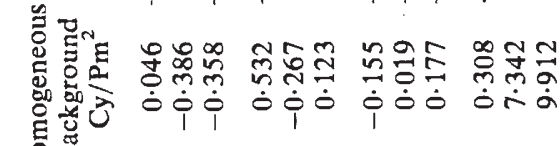

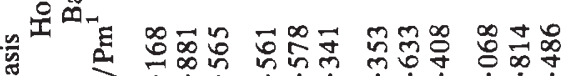

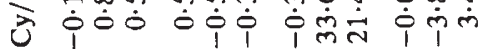

घ

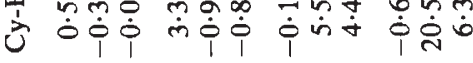
을

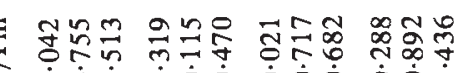
造数 空

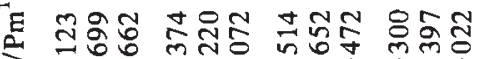

$$
\begin{aligned}
& \text { 向 } \\
& \text { 以NB WNB WNB WNB }
\end{aligned}
$$

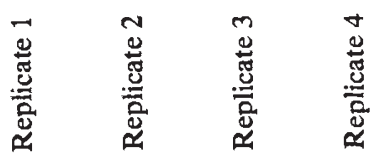




$$
\begin{aligned}
& \text { ৩ }
\end{aligned}
$$

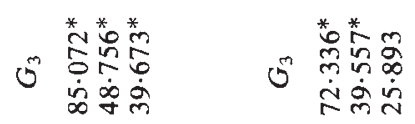

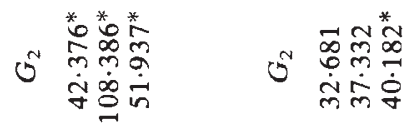

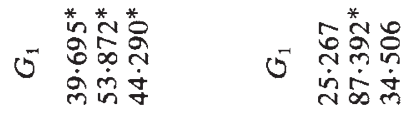

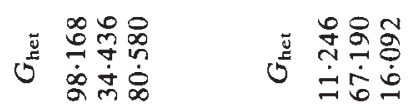

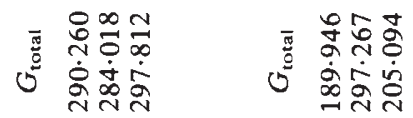

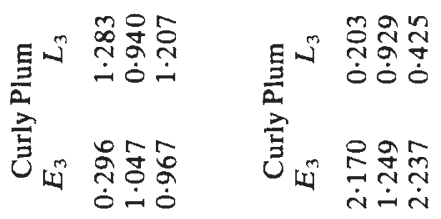

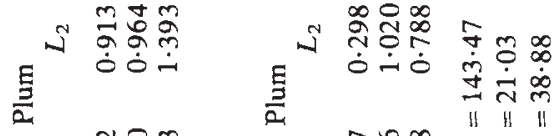

$$
\begin{aligned}
& \text { 山 }
\end{aligned}
$$

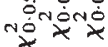

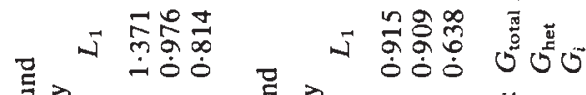

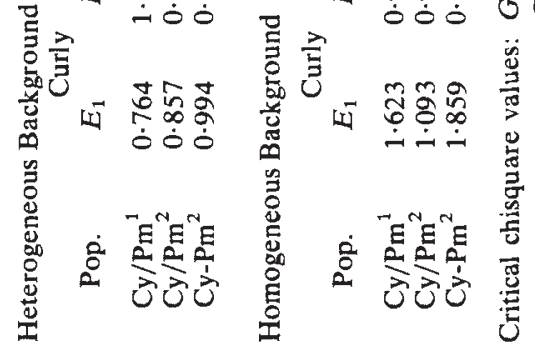


FITNESS IN DROSOPHILA

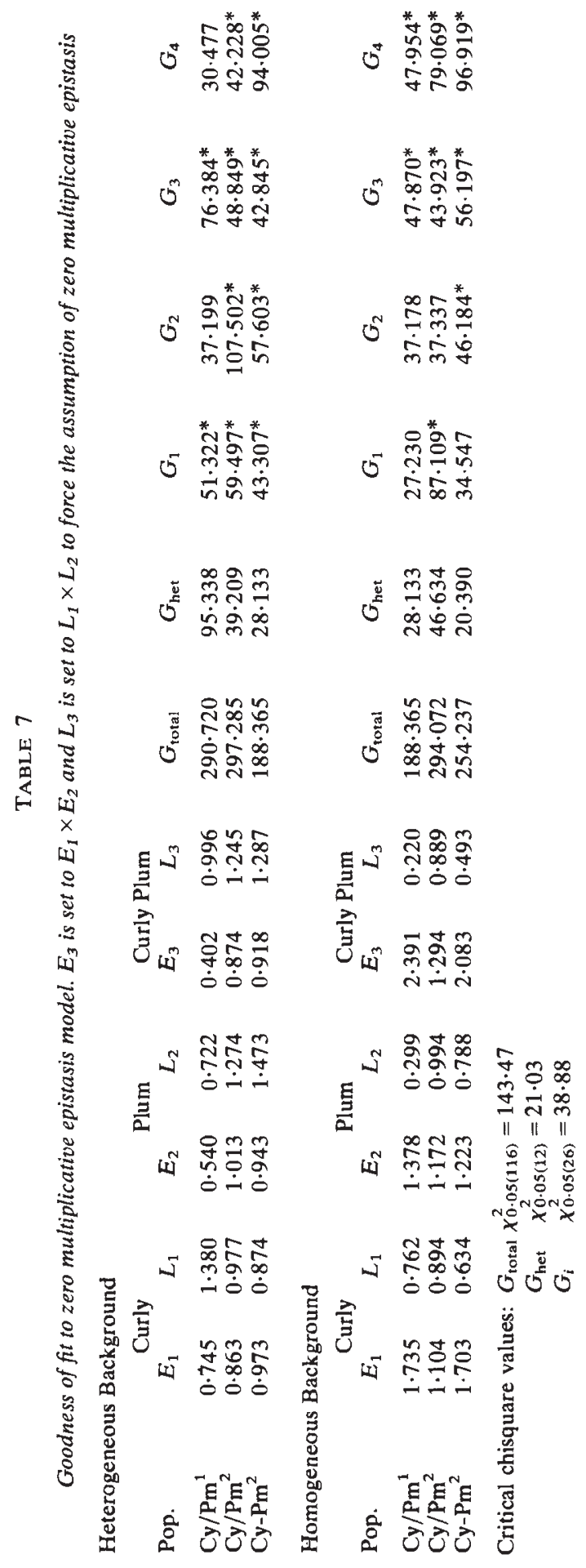


suggest that the epistatic model fits the observations better than the models that lack epistasis.

\section{Discussion}

The $G$ statistics indicate that the two stage model fits the data from the marginal populations well. In the populations where two loci are segregating, the single replicate $G$ statistics generally indicate that the model acceptably fits the data, although some minor deviations are suggested. The observed heterogeneity between replicates underscores this tendency towards deviations from the model. These deviations may occur for two main reasons:

(1) the applied fitness model is only an approximation, and

(2) the statistical model neglects some sources of variation and temporal correlations.

A full description of selection in an experimental Drosophila population requires more detail than used in the present experiments (Bungaard and Christiansen 1972), and the fitness components of the genotypes considered here were investigated in auxiliary experiments to be reported elsewhere (Clark and Feldman, 1981a). In these experiments male mating success varied with genotypic frequencies, which should show up as temporal heterogeneity in the present experiments. Mating type frequencies were ascertained in the auxiliary experiments and random mating was generally observed. No significant meiotic drive was observed. Differences between the sexes in viability were small, but the differences in the fertility components were large. Despite these sex differences, it is not surprising that the model fitted the data because, as Anderson (1969) and Christiansen et al. (1977) demonstrated, the trajectories are closely approximated by setting the fitnesses to the mean of the two sexes. Differences between the two double heterozygotes were seen in viability, female fecundity and male fecundity, but position effects could not be seen in the trajectory data reported here; from table 4 the two estimates of the net fitness of the $\mathrm{CyPm}^{2}$ phenotypes from the $\mathrm{Cy} / \mathrm{Pm}^{2}$ and $\mathrm{Cy}-\mathrm{Pm}^{2}$ populations are very similar.

Stochastic fluctuations in fitness parameters have been neglected in the statistical model which assumes a multinomial sampling distribution. Similarly autocorrelations between generations have been neglected, although the experimental design is likely to cause negative autocorrelations because the females were not virgins at the time of transfer. These simplifications seem unimportant in the marginal populations as the fit of the model is indeed satisfying. In the populations with two loci segregating, both of these effects are amplified due to the presence of rare types in all generations, and to the incomplete information on the linkage disequilibrium. These assumptions are likely to produce both temporal heterogeneity and heterogeneity between replicates.

Environmental heterogeneity may contribute to both temporal heterogeneity and to heterogeneity among replicates. When trajectories were generated based on the estimated fitness values with multinomial sampling (e.g., fig. 8 and 9), it became apparent that there is temporal heterogeneity in the selection coefficients as replicates at times deviated from the model in coordinated fashion. Despite the observed heterogeneities, we feel 
confident in the main conclusion that epistatic interactions are necessary to account for the observed population trajectories and are reflected in the relative goodness of fit of the models.

The standard errors of the fitness components were calculated in the usual way by inverting the information matrix, which was constructed by calculating the second partial derivatives of the likelihood by the method of second differences. The standard errors are then the square roots of the diagonal elements of the inverse of the information matrix (the variancecovariance matrix). If the samples were not multinomial, then this approximation procedure may give spurious results. Furthermore, the likelihood surfaces and the off-diagonal elements of the variance-covariance matrices indicate that the estimators of the early and late components are correlated. The confidence areas of the estimators are plotted graphically based on values of the $G$ statistic calculated for each point in the $E$ - $L$ plane (fig. 10). If a point $(E, L)$ yielded a $G$ less than the 5 per cent critical chisquare value, this meant that the pair of estimators gave a sufficiently good fit to the data. By printing a dot for each point which had a sufficiently small $G$, the computer plotted a 95 per cent confidence area in the $E$ - $L$ parameter space. The method of calculating standard errors from the partial derivatives yields a syrnmetric ellipse for the confidence area, whereas the empirical

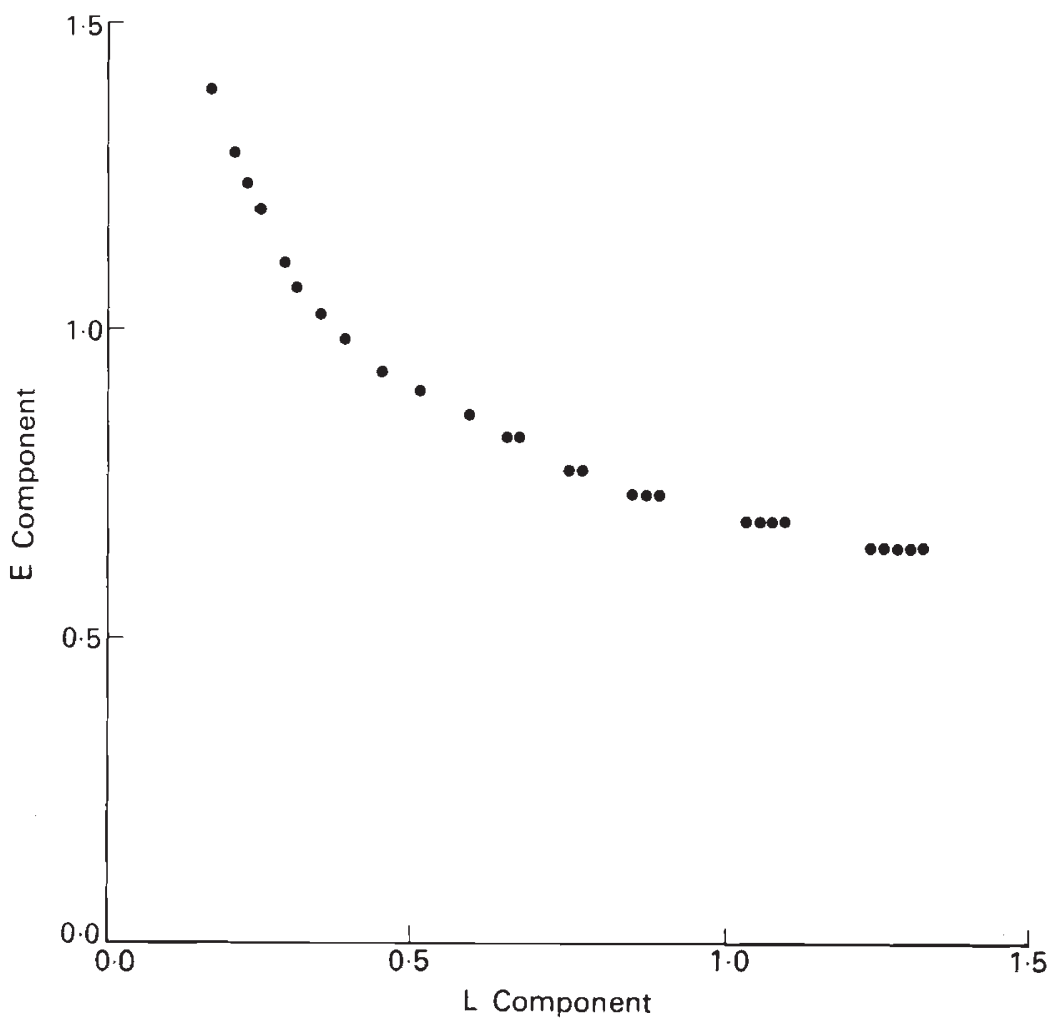

FIG. 10.-Ninety-five per cent confidence area of $E$ and $L$ estimators for homogeneous $\operatorname{Pm} 2$ populations. A dot is printed for each point in the $E-L$ plane which yields a pooled $G$ value less than the critical chisquare value (see text). 
confidence area is far from symmetric. Since the net fitness $W=E \times L$, we would expect a hyperbolic relationship between $E$ and $L$. The narrowness of the confidence area plotted in fig. 10 leads us to conclude that our confidence in the estimate for $W$ is quite sharp (the 95 per cent confidence interval is $0 \cdot 475 \pm 0 \cdot 150$ ), but the two-stage maximum likelihood model fails to resolve the early and late components with the same precision.

With the two locus models there are six dimensional 95 per cent confidence hypervolumes. These were examined in two dimensional slices, and in general the slice whose axes were $E i$ and $L i$ looked similar to fig. 10 . The slices in the $E_{i} E_{j}$ or $L_{i} L_{j}$ planes however yielded more elliptical confidence areas (fig. 11). The general conclusion from these plots is that estimators for the net fitnesses had quite small confidence intervals, but the power to resolve these into components is fairly weak.

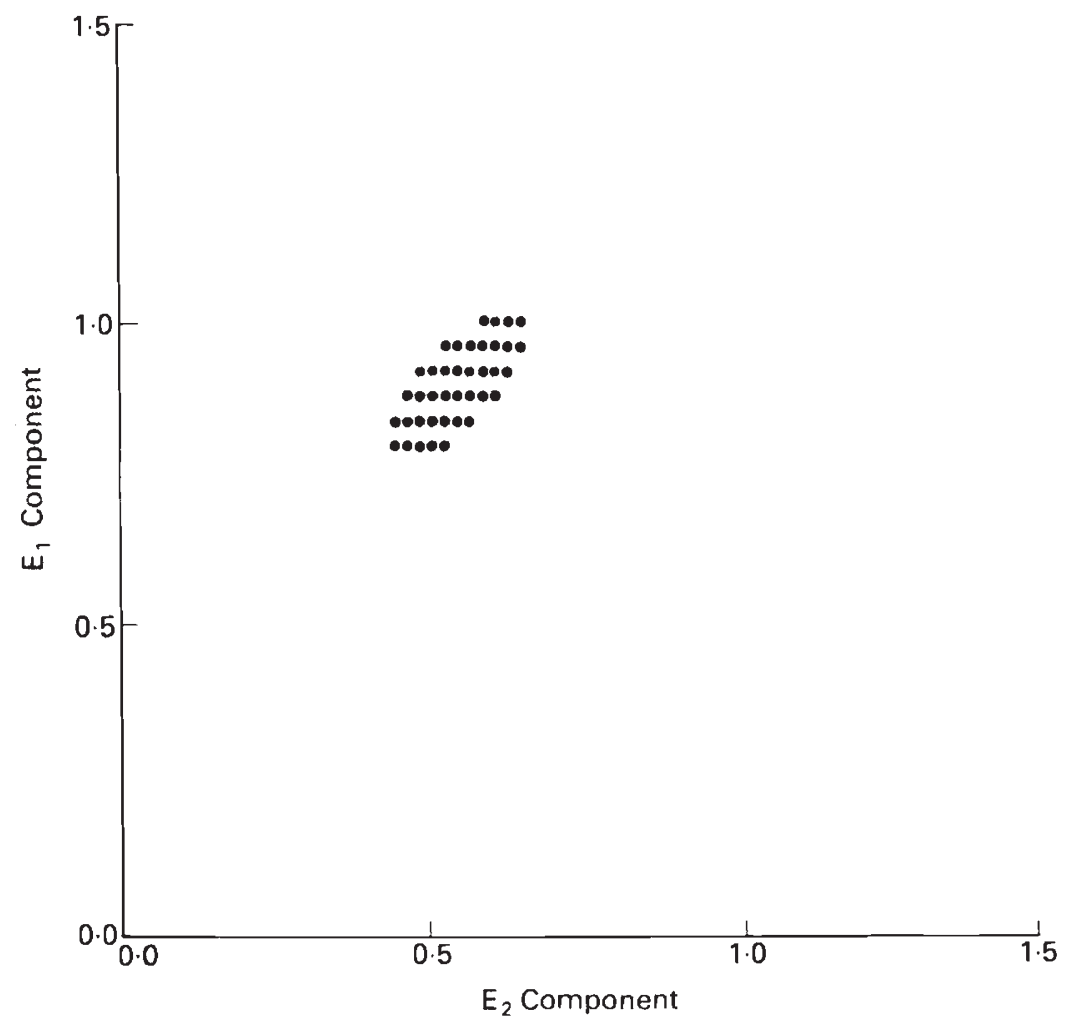

FIG. 11.-Ninety-five per cent confidence area of $E 1$ and $E 2$ in homogeneous $\mathrm{Cy} / \mathrm{Pm} 1$ populations (see text for explanation).

Prout (1965) demonstrated that by sampling a population each generation before selection was completed, an apparent frequency dependence of fitnesses would be observed. By analogy, in a two locus model, if selection is not completed when the population is sampled (i.e., if selection occurs between sampling and the formation of zygotes) then in addition to apparent frequency dependence, apparent epistasis might be observed when it in fact 
is not operating. It is also possible that one might infer a lack of interaction when in fact there is epistasis in the post-sampling component. This is most readily seen by a numerical example (fig. 12 ).

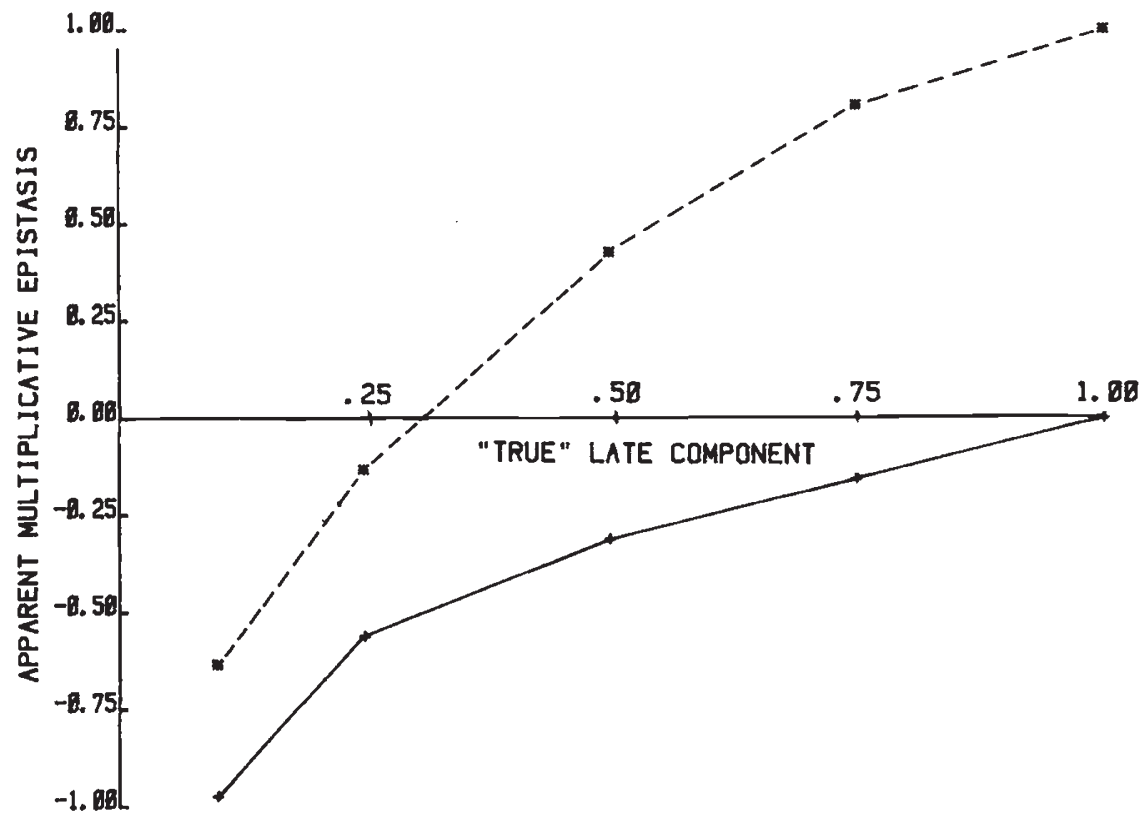

FIG. 12.-Dependence of estimated epistasis on degree of post-sampling selection. The dashed line represents cases in which the net fitnesses were $W_{1}=1, W_{2}=1$, and $W_{3}=2$ (net epistasis $=1 \cdot 0$ ), and the solid line had $W_{1}=W_{2}=W_{3}=1$ (net epistasis $=0 \cdot 0$ ). For each point, gene frequency trajectories were generated with $L_{1}=L_{2}=L_{3}$ set equal to the abscissa value, and the $E$ components adjusted so that net fitnesses remained unchanged. These theoretical trajectories were then used as input data for the maximum likelihood routine, which found the best estimates for $E_{1}, E_{2}$ and $E_{3}$ with the constraint $L_{1}=L_{2}=$ $L_{3}=1$ (i.e., when it was assumed that there was no post-sampling selection operating). The ordinate of each point is then $E_{3} / E_{1} E_{2}-1$.

In general the additive and multiplicative epistasis parameters agree in sign and magnitude for the populations in this study, but this need not be the case in general. For example, in this linked lethal case, if the fitnesses are $w_{1}=0.5, w_{2}=0.5$, and $w_{3}=0.2$ the multiplicative epistasis is -0.2 , while the additive epistasis is $0 \cdot 2$. The way epistasis is defined is thus important in determining its apparent significance. In general the two diallelic locus fitness matrix has four epistasis parameters, while the linked lethal experimental design examined here has just one epistasis parameter. In theory, if all measures of additive epistasis are zero, then the loci will have independent equilibrium behaviour and no linkage disequilibrium, while zero multiplicative epistasis can still generate disequilibrium, provided the linkage is sufficiently tight (Bodmer and Felsenstein, 1967). For fitness components other than viability, there are even more ways to parameterize epistasis, and results may be specific to the chosen parameterization. On the other hand, one experimental observation that is clearly independent of any 
model is the significance of the background effect. The relative fitnesses of $\mathrm{Pm}^{1}$ and $\mathrm{Pm}^{2}$ were fairly similar for both backgrounds, while the Cy fitness changed dramatically (table 3 ).

We have attempted to demonstrate a statistical technique by which gene interactions can be measured in selection trajectories by comparing goodness of fit to epistatic and non-epistatic models. It is reasonable to conclude that the dynamics observed in fig. 5 and 6 were in part a result of fitness epistasis. It is not clear whether the single "locus" effects are closer to being additive or multiplicative, or which measure of epistasis is better in predicting population dynamics. There may also be a third measure of interaction that gives more insight into the dynamics. Epistasis in one component of fitness may not necessarily be sufficient to make a significant difference in the population dynamics of a polymorphism. Epistasis did not generate stable linkage disequilibrium in our experimental populations, because both "loci" did not remain polymorphic. Despite our lack of confidence in the utility of the early and late epistasis components, the possibility that interlocus interactions may occur at different life stages must be acknowledged.

Just as we are unable to accurately assess the relative importance of natural selection in the determination of gene frequencies and distributions, we have a poor understanding of the importance of interactions of selective effects in natural populations. To a large extent this problem can be attributed to the different approaches of the experimental and theoretical science. The hypotheses to be tested concerning selection and epistasis are generated by theory which often demand more than the experimental technology can deliver. The importance of Prout's (1965, 1969, 1971a, $1971 b$ ) contribution to experimental fitness estimation is that it demonstrated a method of partitioning the theoretician's net fitness parameter $W$ into components which were experimentally accessible. In a similar fashion we have attempted to estimate the importance of epistasis in laboratory populations by splitting it into measureable components. The experimental method of selection components analysis using pedigree data is clearly the best suited for this purpose, and interlocus interactions at different stages of the life cycle can be quantified (Clark and Feldman, 1981a). Before there can be significant progress in the assessment of epistasis in natural populations, hypotheses concerning the effects of interlocus interactions in different fitness components must be clearly stated. It remains an interesting theoretical problem to generate these hypotheses by determining the effects of gene interaction at the level of fertility, meiotic drive and sexual selection.

Acknowledgements. - The authors thank R. S. Burton for critical discussion of the manuscript. This work was supported by NIH grants GM28016, GM10452, and NSF grant DEB 77-05747.

\section{REFERENCES}

ANDERSON, W. W. 1969. Selection in experimental populations. I. Lethal genes. Genetics, $62,653-672$.

BAKER, W. K. 1975. Linkage disequilibrium over space and time in natural populations of Drosophila montana. Proc. Nat. Acad. Soc., 72, 4095-4099.

BARKER, J. S. F. 1977. Population genetics of a sex-linked locus in Drosophila melanogaster. I. Linkage disequilibrium and associative overdominance. Hereditas, 85, 169-198. 
BIJLSMA, R. 1978. Polymorphism at the G6PD and 6PGD loci in Drosophila melanogaster. II. Evidence for interaction in fitness. Genet. Res., 31, 227-237.

BIRLEY, A. J. 1974. Multilocus polymorphism and selection in a population of Drosophila melanogaster. I. Linkage disequilibrium on chromosome III. Heredity, 32, 122-127.

BODMER, W. F., AND BODMER, J. G. 1978. Evolution and function of the HLA system. Brit. Med. Bull., 34, 309-316.

BODMER, W. F., AND FELSENSTEIN, J. 1967. Linkage and selection: Theoretical analysis of the deterministic two locus random mating model. Genetics, 57, 237-265.

BUNGAARD, J., AND CHRISTIENSEN, F. B. 1972. Dynamics of polymorphisms. I. Selection components in an experimental population of Drosophila melanogaster. Genetics, 71 , 439-460.

BRNIC, D. 1961. Nonrandom association of inversions in Drosophila pavani. Genetics, 46 , 401-406.

CANNON, G. B. 1963. The effects of natural selection on linkage disequilibrium and relative fitness in experimental populations of Drosophila melanogaster. Genetics, 48, 1201-1216.

CHARLESWORTH, B., CHARLESWORTH, D., LOUKAS, M., AND MORGAN, K. 1979. A study of linkage disequilibrium in British populations of Drosophila subobscura. Genetics, 92, 983-994.

CHRISTIANSEN, F. B., BUNGAARD, J., AND BARKER, J. S. F. 1977. On the structure of fitness estimates under post-observational selection. Evolution, 31, 843-853.

CLARK, A. G., AND FELDMAN, M. W. 1981 $a$, "The estimation of epistasis in components of fitness in experimental populations of Drosophila melanogaster. II. Assessment of meiotic drive, viability, fecundity and sexual selection", Heredity, 46,

CLARK, A. G., AND FELDMAN, M. W. $1981 b$, Disequilibrium between linked inversions: Another hypothesis. Heredity, 46,

ClEGG, M. T., KIDWELl, J. F. AND HORCH, C. R. 1980. Dynamics of correlated genetic systems. V. Rate of decay of linkage disequilibria in experimental populations of Drosophila melanogaster. Genetics, 94, 217-234.

CLEGG, M. T., KIDWELl, J. F. AND KIDWELL, M. G. 1978. Dynamics of correlated genetic systems. III. Behavior of chromosome segments under lethal selection. Genetics, 48 , 95-106.

FELDMAN, M. W., AND CROW, J. F. 1970. On quasilinkage equilibrium and the fundamental theorem of natural selection. Theor. Pop. Biol., 1, 371-391.

FR YDENBERG, O. 1963. Population studies of a lethal mutant in Drosophila melanogaster. I. Behavior in populations with discrete generations. Hereditas, 50, 89-116.

KARLIN, S. 1975. General two locus selection models: Some objectives, results and interpretations. Theor. Pop. Biol., 7, 364-398.

KIMURA, M. 1956. A model of a genetic system which leads to closer linkage by natural selection. Evolution, 10, 278-281.

LEVITAN, M. 1955. Studies of linkage in populations. I. Associations of second chromosome inversions in Drosophila robusta. Evolution, 9,62-74;1958. Nonrandom associations of inversions. Cold Spring Harbor Symp. Quant. Biol., 23, 251-268.

LEWONTIN, R. C. AND KOJIMA, K. 1960. The evolutionary dynamics of complex polymorphism. Evolution, 14, 458-472.

LEWONTIN, R. C. AND WHITE, M. J. D. 1960. Interactions between inversion polymorphisms of two chromosome pairs in the grasshopper Moraba scurra. Evolution, 14, 116-129.

LOUKAS, M. C., AND KRIMBAS, B, 1975. The genetics of Drosophila subobscura populations V. A study of linkage disequilibrium in natural populations between genes and inversions of the E chromosome. Genetics, 80, 331-347.

LOUKAS, M. C., KRIMBAS, B., AND VERGINI, Y. 1979. The genetics of Drosophila subobscura populations. IX. Studies on linkage disequilibrium in four natural populations. Genetics, 93, 497-523.

MARINKOVIC, D., AND AYALA, F. J, 1975a. Fitness of allozyme variants in Drosophila pseudoobscura. I. Selection at the Pgm-1 and $\mathrm{Me}-2$ loci. Genetics, 79, 85-95; $1975 \mathrm{~b}$. Fitness of allozyme variants in Drosophila pseudoobscura. II. Selection at the Est-5, Odh, and Mdh-2 loci. Genet. Res., 24, 137-149.

MARTIN, J. 1962. Interrelation of inversion systems in the midge Chrionomus intertinctus. I. A sex-linked inversion. Aust. J. Biol. Sci., 15, 666-673.

MATHER, W. B. 1961. Chromosomal polymorphism in Drosophila rubida. Genetics, 46, 799-810.

PARSONS, P. A. 1963. Complex polymorphisms where coupling and repulsion double heterozygote viabilities differ. Heredity, 18, 369-374. 
PRAKASH, S. 1974. Gene differences between the sex ratio and standard gene arrangements of the $\mathrm{X}$ chromosome and linkage disequilibrium between loci in the standard gene arrangement of the X chromosome in Drosophila pseudoobscura. Genetics, 77, 795-804; 1977. Gene differences between third chromosome inversions of Drosophila pseudoobscura. Genetics, 84, 787-790.

PRAKASH, S., AND LEWONTIN, R. C. 1968. A molecular approach to the study of genic heterozygosity in natural populations. III. Direct evidence of coadaptation in gene arrangement of Drosophila. Proc. Natl. Acad. Sci., 59, 398-405; 1971. A molecular approach to the study of genic heterozygosity in natural populations. V. Further direct evidence of coadaptation of inversions of Drosophila. Genetics, 69, 405-408.

PROUT, T. 1965. The estimation of fitnesses from genotypic frequencies. Evolution, 19, $546-551 ; 1969$. The estimation of fitnesses from population data. Genetics, 63, 949-967; $1971 a$. The relation between fitness components and population prediction in Drosophila. I. Estimation of fitness components. Genetics, 68,127-149;1971 b. The relation between fitness components and population prediction in Drosophila. II. Population prediction. Genetics 68, 151-167.

STALKER, H. D. 1960. Chromosomal polymorphism in Drosophila paramelanica. Genetics, $45,95-114$.

WEIR, B. S. AND COCKERHAM, C. C. 1978. Testing hypotheses about linkage disequilibrium with multiple alleles. Genetics, 88, 633-642.

WILSON, J. 1968. Experimental determination of fitness interactions in Drosophila melanogaster by the method of marginal populations. Genetics 59, 501-511; 1972. Experimentally determined two locus fitnesses of Drosophila melanogaster males. Genetics, 70 , 445-455.

ZOUROS, E., AND KRIMBAS, C. B. 1973. Evidence for linkage disequilibrium maintained by selection in two natural populations of Drosophila subobscura. Genetics, 73, 659-674. 